\title{
Hyperkalaemia, cardiac arrhythmias, and cerebral lesions in high risk neonates
}

\author{
D SHORTLAND, ${ }^{*} \mathrm{~J}$ Q TROUNCE, $\dagger$ AND M I LEVENE* \\ Departments of Child Health, Leicester Royal Infirmary* and Nottingham City Hospital ${ }^{*}$
}

SUMmARY The case notes of 20 infants with hyperkalaemia (defined as two successive serum potassium measurements of $>7.5 \mathrm{mmol} / \mathrm{l}$ ) were reviewed. The incidence of hyperkalaemia was also looked at in an unselected population of 200 low birthweight infants. The mean gestational age of the 20 affected infants was 29 weeks and the mean birth weight $1235 \mathrm{~g}$. The incidence of hyperkalaemia in the cohort of 200 infants weighing less than $1500 \mathrm{~g}$ at birth was $3 \cdot 5 \%$. Hyperkalaemia was associated with a high incidence of cardiac arrhythmia $(60 \%)$, impaired renal function $(50 \%)$, and changes on cerebral ultrasonography $(88 \%)$. Hyperkalaemia responds slowly to conventional treatment with dextrose, insulin, and exchange resins. There is a close temporal relation in some infants between hyperkalaemia and cardiac arrhythmias and periventricular leukomalacia, suggesting a causal association.

There are little published data on the incidence and consequences of neonatal hyperkalaemia. It is known to cause cardiac arrythmias, ${ }^{1-4}$ and recent evidence suggests that increased potassium concentrations in cerebrospinal fluid or brain parenchyma may cause spasm of the intracerebral arteries. ${ }^{5}$ Both these factors could impair the neonatal cerebral circulation.

We looked at the incidence of hyperkalaemia in an unselected population of very low birthweight infants $(n=200)$, and reviewed the case notes of 20 infants known to have had hyperkalaemia to estimate the incidence of cardiac arrhythmia, renal impairment, and cerebral abnormality.

\section{Methods}

A computer search for cases of hyperkalaemia among all admissions to the neonatal unit from April 1983 until May 1986 was undertaken. Hyperkalaemia was defined as two sequential serum potassium measurements of $7.5 \mathrm{mmol} / \mathrm{l}$ or more in non-haemolysed samples. In an earlier study at the Leicester Royal Infirmary and Nottingham City Hospital neonatal units 200 consecutive very low birthweight infants had had their neonatal complications documented prospectively. Serum potassium concentrations were recorded and from these data we calculated the incidence of hyperkalaemia in this group of very low birthweight infants.
The biochemical data were obtained in all the infants with hyperkalaemia. High serum urea or creatinine concentrations were defined as those above the normal range given in published reports. ${ }^{67}$ Twelve hourly arterial $\mathrm{pH}$ measurements before and during the hyperkalaemic episodes were recorded to assess the degree of acidosis. We assessed the duration and severity of hyperkalaemia by calculating in $\mathrm{mm}^{2}$ the area under the curve that

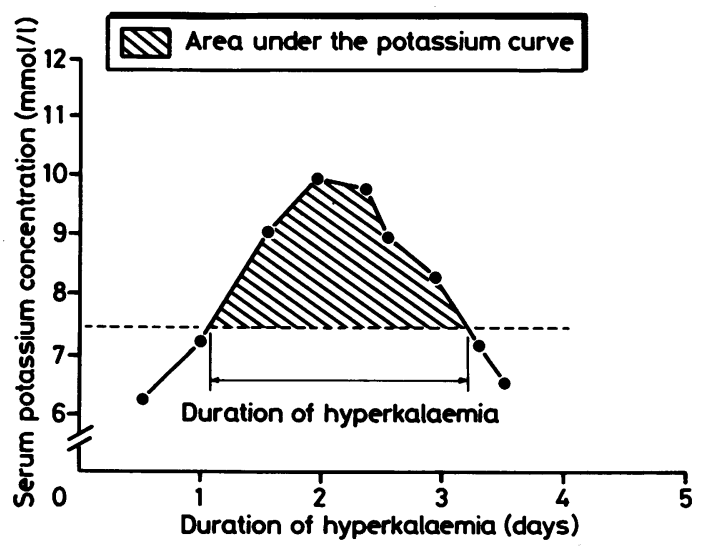

Fig 1 Method of calculating duration and severity of hyperkalaemia (serum potassium concentration of $>7.5$ mmol/l). 
1140 Shortland, Trounce, and Levene

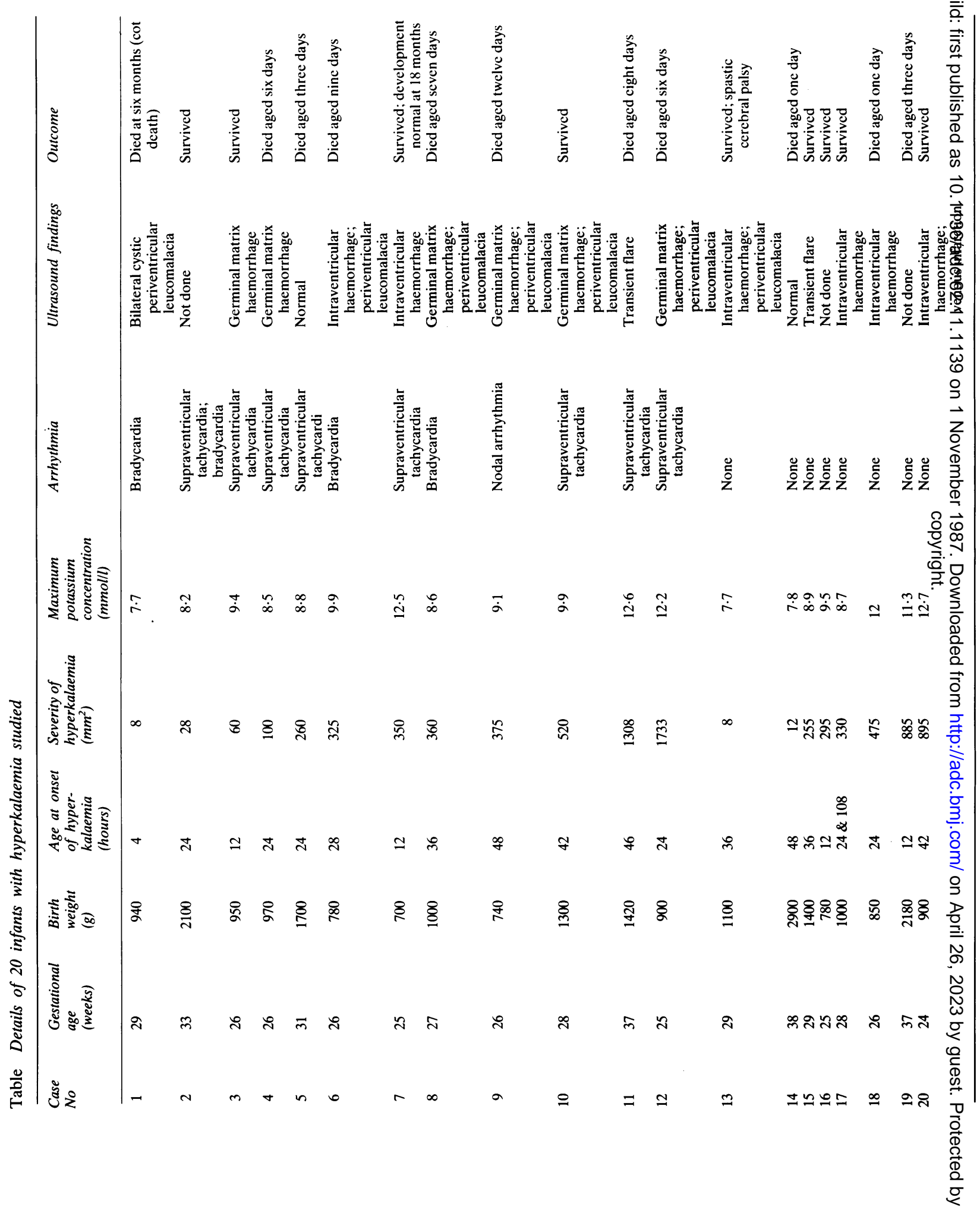


related serum potassium concentration to time (fig 1). This method has been previously used to assess the severity of acidosis. ${ }^{8}$ The number of episodes of cardiac arrhythmia and the treatment of hyperkalaemia were recorded and cerebral ultrasound scans of the hyperkalaemic infants were also reviewed.

\section{Results}

Seven of the 200 very low birthweight infants in the previously studied cohort developed hyperkalaemia in the neonatal period, ${ }^{9}$ an incidence of $3.5 \%$. We report the results from an additional 13 hyperkalaemic infants.

The mean birth weight of the 20 infants was $1230 \mathrm{~g}$ and the mean gestational age 29 weeks. In all the infants the hyperkalaemia became apparent within 48 hours of birth, and one infant had a second episode during the fifth day of life. The table gives details of the data on the infants. No infants had had prolonged seizures, and blood transfusions were not given in the first 48 hours of life, so these two possible causes of hyperkalaemia could be disregarded.

Serum urea concentrations were available for all 20 infants and creatinine concentrations were also available for 11 infants, of which five were abnormal. We used creatinine concentrations as an index of renal function when available, and the less reliable urea measurements in the rest. In the nine infants in whom only urea concentrations were available, five were above the normal range.

Arterial $\mathrm{pH}$ estimations were available before and during the hyperkalaemic episode in 10 infants, and these showed that there was no obvious trend towards acidosis when the serum potassium concentrations were at their highest.

Cardiac arrhythmias occurred in $12(60 \%)$ of the infants; the most common was supraventricular tachycardia $(n=7)$; three became bradycardic; one developed a nodal rhythm; and one had both supraventricular tachycardia and. bradycardia. In four infants the arrhythmia preceded the diagnosis of hyperkalaemia. Five infants with cardiac arrhythmias had raised serum urea or creatinine concentrations, and therefore possible renal compromise. The mean peak potassium concentration in the group with arrhythmias was $9.8 \mathrm{mmol} / \mathrm{l}$. This is the same as that found in the infants who did not have arrhythmias. The mean area under the curve in infants with an arrhythmia was $452 \mathrm{~mm}^{2}$ compared with $394 \mathrm{~mm}^{2}$ in those with no arrhythmia; this difference was not significant. In some infants with cardiac arrhythmia the blood pressure had been recorded continuously. In each infant with supraventricular tachycardia or nodal rhythm in whom continuous recordings of blood pressure were available there was a definite fall during the arrhythmia (fig 2).

The results of cerebral ultrasound scans were available in 17 of the infants. In two the scan remained normal throughout the neonatal period. The remaining $15(88 \%)$, however, showed some abnormality; 11 had germinal matrix haemorrhage or intraventricular haemorrhage, and seven periventricular leucomalacia with or without germinal matrix haemorrhage or intraventricular haemorrhage. In a further two infants there were transient echodense areas in the parenchyma but these all resolved within two weeks of birth. In five infants with periventricular leucomalacia we determined the association between the diagnosis of hyperkalaemia and the onset of the lesion seen on the ultrasound scan. In three, parenchymal echodensity was first detected within 48 hours of the raised serum potassium concentration, and in another the next scan (performed four days after the onset of hyperkalaemia) showed periventricular leucomalacia for the first time. In one infant periventricular leucomalacia preceded the hyperkalaemia, and in three the ultrasound evidence of periventricular leucomalacia was seen within 24 hours of cardiac arrhythmia. The infant whose blood pressure trace is shown in fig 2 developed parenchymal echodensity within 12 hours of the documented arrhythmia. In only one infant was periventricular leucomalacia evident before the diagnosis of hyperkalaemia.

The hyperkalaemia was treated with insulin and infusion of $10 \%$ dextrose; calcium resonium enemas were given every four hours. Episodes of arrhythmia

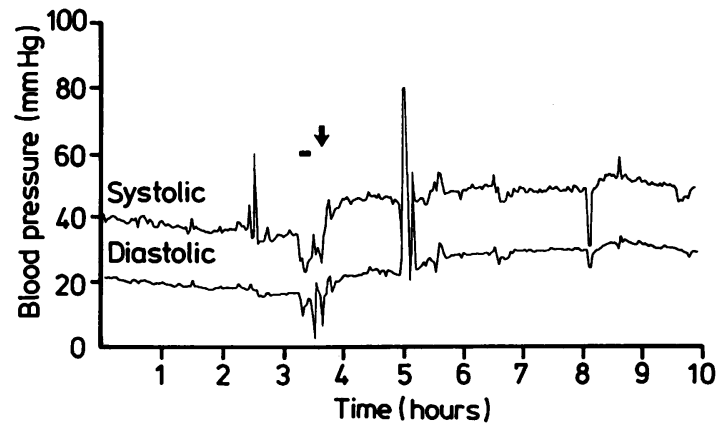

Fig 2 Effects of supraventricular tachycardia on blood pressure in infant of 27 weeks' gestation when 24 hours old. Bar $=$ duration of arrhythmia, terminated with $10 \%$ calcium gluconate (arrow). Systolic and diastolic blood pressures were recorded on disc every two minutes and displayed as a rolling average. ${ }^{14}$ 
were treated with bolus injections of $0 \cdot 2-0.5 \mathrm{ml}$ of $10 \%$ calcium gluconate and cardioversion was not used. Case 10 (table) was treated by peritoneal dialysis.

\section{Discussion}

Despite the relatively high incidence of hyperkalaemia in the two neonatal units studied little seems to have been written about the condition in neonates. An infant is most likely to have raised serum potassium concentrations within the first 48 hours of life. At this time he or she will be receiving minimal amounts of oral or intravenous potassium, and so the likely cause of the condition is either leakage of intracellular potassium or diminished renal excretion. Potassium is predominantly an intracellular cation and any factor which causes it to move out of the cells (for example, acidosis) will result in an increased serum concentration. The rate of potassium excretion depends on the concentration gradient between the distal renal tubular cells and the luminal potassium concentration. ${ }^{10}$ It follows that any factor increasing the intracellular potassium concentration (for example, insulin) or increasing luminal urine flow rates (for example, diuretics) will enhance potassium excretion.

The most important function of potassium is to maintain the normal membrane resting potential. An increase in extracellular potassium concentration will lower the resting potential and therefore a stimulus of lesser intensity will evoke an action potential. This will increase the 'excitability' of the cell membrane. An action potential is produced by the opening of sodium channels, thereby permitting the rapid entry of sodium into the cells. When potassium concentrations are extremely high these channels are inactivated and the stimulus cannot produce an electrical response. ${ }^{11}$ This may account for the bradyarrhythmias associated with hyperkalaemia.

Therefore it is not surprising that so many of the infants $(60 \%)$ developed cardiac arrhythmias. Two kinds of arrhythmia predominated in our study, supraventricular tachycardia and sinus bradycardia, but many different electrocardiographic changes have been previously reported. ${ }^{2-4}$

Hyperkalaemia seems primarily to affect very low birthweight infants and review of the case notes of these infants showed that the most likely to suffer from it are those with other problems. Guignard et al showed that renal function was impaired in infants with idiopathic respiratory distress syndrome and that the degree of impairment correlated with the severity of the respiratory illness. ${ }^{12}$ They postulated that the hypoxaemia, hypotension, or intermittent positive pressure ventilation may have been responsible. Measurements of serum urea and creatinine concentrations were available for all the infants, and $10(50 \%)$ of them had evidence of renal impairment. This was probably the cause of their hyperkalaemia, but we were unable to identify the cause in the remainder. There may have been a reduction in renal blood flow (and therefore in distal tubular luminal urine flow) that was sufficient to impair potassium excretion but not sufficient to be reflected in raised urea and creatinine concentrations. It is more likely, however, that there was a redistribution of potassium from the intracellular to the extracellular compartments. Acidosis causes such shifts in potassium, but we did not see appreciable decreases in arterial $\mathrm{pH}$ during periods of hyperkalaemia.

Edvinsson et al showed that human cerebral arteries in vitro contract when bathed by a potassium concentration of more than $10 \mathrm{mmol} / \mathrm{l} .{ }^{5}$ They postulated that this may be partly responsible for periventricular ischaemia in neonates. Abnormalities on ultrasound scan were seen in 15 of the 17 infants $(88 \%)$, of whom eight $(54 \%)$ had parenchymal changes. In a previous study of 200 very low birthweight infants parenchymal lesions were seen in only $14 \% .{ }^{9}$ In the present study, germinal matrixe haemorrhage or intraventricular haemorrhage was seen in $70 \%$ of the infants. The close temporal relation between hyperkalaemia and periventricular leucomalacia that we found provides good circumstantial evidence of a direct association between them. In addition, three infants showed the first signs of periventricular leucomalacia within 24 hours of cardiac arrhythmia. In the case illustrated in fig 2 there was a period of acute hypotension lasting for over 20 minutes 12 hours before the first ultrasound changes of periventricular leucomalacia appeared, which supports a direct causal association between arrhythmia and cerebral infarction.

Our current policy is to treat any infant with a serum potassium concentration of $>7.5 \mathrm{mmol} / \mathrm{l}$, or a concentration of $6.5-7.5 \mathrm{mmol} / \mathrm{l}$ if it is associated with electrocardiographic abnormalities. Cardiac arrhythmias are treated with a bolus dose of $10 \%$ calcium gluconate intravenously. In the present study 13 of the infants were treated with insulin and dextrose infusions and calcium resonium exchange enemas. In these infants the mean duration of the hyperkalaemic episode was 47 hours. Because potassium concentrations decline slowly with conventional treatment we have begun to evaluate peritoneal dialysis; to date five infants have been treated in this way. Setzer et al reported the successful treatment of neonatal hyperkalaemia by an exchange transfusion of red blood cells washed in 
saline. ${ }^{13}$ Both these methods require further evaluation.

Hyperkalaemia may cause cerebral damage by reducing cerebral blood flow during episodes of cardiac arrhythmia or by inducing arteriolar spasm. In the absence of evidence to the contrary, we recommend that hyperkalaemia should be treated aggressively. Serum potassium concentrations should be checked regularly during the first 48 hours of life in very low birthweight infants, particularly in those with other problems.

Drs Shortland and Trounce were supported by the Spastics Society.

\section{References}

1 Fisch C. Relations of clectrolyte disturbances to cardiac arrhythmias. Circulation 1973;47:408-19.

2 Cohen HC, Rosen KM. Pick A. Disorders of impulse conduction and impulse formation caused by hyperkalaemia in man. Am Heart J 1975;89:501-9.

${ }^{3}$ Herscher E, Berman W, Friedman Z, Whitman V. Left bundle branch block due to hyperkalacmia in premature infants. J Pediatr 1979;95:654-6.

4 Weidner NJ, Gaum WE, Te-Chuan C. Kayslan S Hyperkalacmia-clectrocardiographic abnormalitics. J Pediatr 1978:93:462-4.

${ }^{5}$ Edvinsson L, Lou HC. Tuede K. On the pathogenesis of regional cerebral ischaemia in intracranial hemorrhage: a causal influence of potassium? Pediatr Res 1986:20:478-80.
"Avery ME, Tacusch HW. Schaffer's diseases of the newborn. 5th ed. Philadelphia: W B Saunders Co.. 1984.

${ }^{7}$ Rudd PT. Hughes EA. Placzek MM. Hodes DT. Reference ranges for plasma creatinine during the first month of life. Arch Dis Child 1983:58:212-5.

* Skouteli HN. Dubowitz LMS. Levene MI. Miller G. Predictors for survival and normal neurodevelopmental outcome of infants weighing less than 1001 grams at birth. Dev Med Child Neurol 1985:27:588-95.

${ }^{y}$ Trounce JQ, Rutter N. Levene MI. Periventricular leucomalacia and intraventricular hacmorrhage in the preterm neonate. Arch Dis Child 1986;61:1196-202.

11' Good DW. Wright FS. Luminal influences on potassium secretion: sodium concentration and fluid flow rate. Am J Physiol 1979;236:192-204.

" Hodgkin AL, Horowicz P. The influence of potassium and chloride ions on the membrane potential of single muscle fibres. J Physiol 1959;148:127-60

12 Guignard JP, Torrado A, Mazouni SM, Gautier E. Renal function in respiratory distress syndrome. $J$ Pediatr 1976:88: $845-50$.

1.3 Setzer ES, Ahmed F, Goldberg RN. et al. Exchange transfusion using washed red blood cells reconstituted with fresh frozen plasma for treatment of severe hyperkalaemia in the neonate. J Pediatr 1984;104:443-6.

it Diprose GK, Evans DH, Levene MI. A microcomputer monitoring and data-acquisition system for intensive care units. J Med Eng Technol 1985:9:80-4.

Correspondence to Dr MI Levene, Neonatal Unit. Department of Child Health. Leicester University School of Medicine, Leicester.

Received 8 Junc 1987 